\title{
Capacitive effects in quasi-steady-state voltage and lifetime measurements of silicon devices
}

\author{
A. Cuevas ${ }^{\text {a) }}$ \\ Department of Engineering, Faculty of Engineering and Information Technology, The Australian National \\ University, Canberra ACT 0200, Australia \\ F. Recart \\ Teknologia Mikroelektronikoaren Institutua, Euskal Herriko Unibertsitatea, Urkixo Zumardia, \\ Bilbao 48013, Spain
}

(Received 29 April 2005; accepted 17 June 2005; published online 11 October 2005)

\begin{abstract}
When measuring $I-V$ characteristics and carrier lifetimes in quasi-steady-state (QSS) conditions, it is important to consider the time dependence of the charge due to excess carriers within the device. This paper shows that the space-charge region present in $p n$-junction devices and in many lifetime test structures can produce a significant capacitive effect when measuring the low voltage and low carrier density range of QSS $I-V$ curves. Both computer modeling and experiments show that the junction capacitance is particularly significant in the case of low-resistivity silicon wafers, but it can also be noticeable in intermediate and high-resistivity samples. The paper demonstrates that the static $I-V$ characteristics can be accurately reconstructed using a simple analytical model for the space-charge region. It thus fills a gap in the understanding of the low injection range of QSS voltage and lifetime measurements. (C) 2005 American Institute of Physics.
\end{abstract}

[DOI: $10.1063 / 1.2073973$ ]

\section{INTRODUCTION}

The measurement of the open-circuit voltage versus illumination intensity in the quasi-steady-state ${ }^{1}\left(\mathrm{QSSV}_{\text {oc }}\right)$ is a fast and convenient method for determining the static diode characteristics of solar cells. It is, at the device level, an analog to the photoconductance ${ }^{2}$ (QSSPC) technique widely used to measure the carrier lifetime as a function of the excess carrier density. Since voltage can be translated into excess carrier density, the QSSV $_{\text {oc }}$ method can, in its own right, be used to characterize carrier lifetime, and vice versa. Both methods are inherently based on changing the injection level as a function of time. Commonly, this is achieved with a photographic flash or a cluster of lightemitting diodes (LED) whose brightness fades with a characteristic time constant of one to several milliseconds. Such slow dimming is usually enough to resemble very closely the desired static conditions for most silicon solar cells and over their most important operating regime, that is, for voltages and carrier densities in the vicinity of the maximum power point.

Nevertheless, transient effects can still be present in QSS measurements. The extended analysis proposed by Nagel et $a l .{ }^{3}$ for QSSPC and Kerr et al. ${ }^{4}$ for QSSV $_{\text {oc }}$ can deal with situations where the carrier lifetime is greater than the dimming constant of the light source, conditions that can be more properly labeled as quasitransient. The success of those extended analyses has led most people to presume that they are completely general, ignoring that, as most analytical models, they have limitations. As lifetime measurement techniques become more sensitive, reaching excess carrier den-

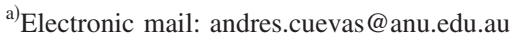

sities in the $10^{11} \mathrm{~cm}^{-3}$ range for photoluminescence, ${ }^{5}$ and even lower for $\mathrm{QSSV}_{\text {oc }}$, some of these limitations are becoming apparent. In particular, the capacitive effects associated with the space-charge region (SCR) of the $p n$ junction can significantly distort the measured $I-V$ curves. These SCR effects were ignored in the previous QSS models, which were restricted to considering the rate of change of the excess carrier charge in the base region. Conversely, junction capacitive effects have historically been a major preoccupation when performing experiments in which brusque temporal changes occur, such as in diode switching. For example, in the open-circuit voltage decay (OCVD) technique, junction capacitance frequently dominates a wide range of the observable voltage transient. ${ }^{6}$

Although not as exposed to it, the $\mathrm{QSSV}_{\mathrm{oc}}$ technique is not totally impervious to the junction capacitance. As shown in this paper, the space-charge region contributes with a time-dependent capacitive effect that, although usually negligible in QSS photoconductance measurements, is often dominant in the lower injection level of the $\mathrm{QSSV}_{\text {oc }}$ curves. A good understanding of this effect and the ability to model it are essential to extract relevant information about SCR recombination, leakage, and shunting losses, which are endemic in the low voltage range of the $I$ - $V$ characteristics.

In addition, the SCR has been shown to distort the measurement of the excess photoconductance in photoconductance decay ${ }^{7}$ (PCD) and QSSPC $^{8}$ lifetime measurements, through the so-called depletion region modulation (DRM) effect. $^{9,10}$ The distinction between DRM, which has no role in neither voltage nor photoluminescence measurements, and SCR capacitance is also clarified in this paper. 


\section{EXCESS CARRIER CHARGE AND RECOMBINATION IN SOLAR CELLS}

Upon excitation, semiconductors develop electrons and holes in excess over their equilibrium densities. The integrated excess carrier density within a particular region, multiplied by the elementary charge $q$, is commonly referred to as the excess carrier charge. For example, in a $p$-type base region,

$$
Q_{B}(t)=q \int_{0}^{W_{B}} \Delta n_{B} d x .
$$

Although the electron charge is negative, we have not included a minus sign in the definition of $Q_{B}$. Note that charge neutrality still applies to the base region thanks to the coexisting excess hole concentration, $\Delta p \approx \Delta n$. In response to a light-induced photogeneration rate $G_{T}$, excess carriers recombine at a rate $U_{T}$, reduce or build up their numbers (that is, modify the excess carrier charge $d Q_{T} / d t$ ), or flow out of the device, $J$. These mechanisms are perfectly balanced, which can be expressed mathematically as

$$
q U_{T}=q G_{T}-\frac{d Q_{T}}{d t}-J .
$$

This equation, where $Q_{T}$ is the total carrier charge "stored" in the different regions of the device, constitutes the central formulation of the charge control model, ${ }^{11,12}$ and has been amply documented in classic semiconductor device texts. ${ }^{13}$ In a typical solar cell, the total recombination rate $U_{T}$ is composed of recombination in the bulk of the wafer, recombination within the space-charge region, and recombination at the surface regions. Recombination within the spacecharge region is frequently masked by leakage and shunting losses, and rarely follows simple theoretical models; its value is expressed below by the term $J_{\mathrm{SCR}}$. Recombination at the surfaces is represented in Eq. (3) by means of the electron current densities $J_{\text {Front }}$ and $J_{\text {Rear }}$. In silicon solar cells, the surfaces are typically occupied by $n^{+}$and $p^{+}$diffusions, commonly referred to as the emitter and back surface field (BSF) regions. In devices not having a BSF, the current $J_{\text {Rear }}$ takes the form of a surface recombination velocity $S_{\text {rear }}$ multiplied by the excess carrier concentration; if $S_{\text {rear }}$ is very high, the diffusion of electrons towards the surface limits the upper value of $J_{\text {Rear }}$. Samples used for lifetime testing are usually symmetrical, having identical emitter regions or surface recombination velocities on both the front and rear surfaces. Finally, recombination in the volume of the wafer is given by the ratio between the base charge (that is, the excess carrier density profile in the base) and the minority carrier lifetime (electrons in a $p$-type base, for example) $\tau_{n B}$. The total recombination rate $U_{T}$ can then be written as

$$
q U_{T}=\frac{Q_{B}}{\tau_{n B}}+J_{\mathrm{SCR}}+J_{\text {Front }}+J_{\text {Rear }} .
$$

For simplicity, we have assumed that the minority carrier lifetime in the base region $\tau_{\mathrm{nB}}$ is spatially constant. Alternatively, $\tau_{\mathrm{nB}}$ could be viewed as the average lifetime within the base. From a practical point of view, it is useful to define a base-centric effective lifetime that represents all recombina- tions within the device as the ratio between the base charge and $\tau_{\text {eff }}$,

$$
\begin{aligned}
& q U_{T} \equiv \frac{Q_{B}}{\tau_{e f f}}, \\
& \frac{1}{\tau_{\text {eff }}}=\frac{1}{\tau_{\mathrm{nB}}}+\frac{1}{Q_{B}}\left(J_{\mathrm{SCR}}+J_{\text {Front }}+J_{\text {Rear }}\right) .
\end{aligned}
$$

The effective lifetime is a complicated parameter that encompasses all the different carrier losses within the device. It is, in principle, possible to extract information on each of those mechanisms from $\tau_{\text {eff }}$, but this is no trivial task. Measuring the effective lifetime over a broad range of carrier injection levels provides additional information that can be exploited to separate them, but even in this case, it is necessary to carefully consider the possible contributions from the remaining recombination terms. ${ }^{14,15}$

Once the effective lifetime has been defined, and after noting that the emitter charge term $d Q_{E} / d t$ can usually be neglected, ${ }^{12}$ Eq. (2) can be rewritten as

$$
\frac{Q_{B}}{\tau_{\text {eff }}}=q G_{T}-\frac{d Q_{B}}{d t}-\frac{d Q_{\mathrm{SCR}}}{d t}-J .
$$

This equation displays the different methods that may be used to measure $\tau_{\text {eff }}$. First and foremost, the excess carrier density (that is, $Q_{B}$ ) needs to be measured in all cases. Next, one can choose to measure: (a) $Q_{B}$ under illumination (typically a steady state or slowly varying illumination $G_{T}$ $=N_{\text {ph }} f_{\text {abs }}$, where $N_{\text {ph }}$ is the incident photon flux and $f_{\text {abs }}$ is the fraction of those photons absorbed within the silicon wafer), (b) the rate of change of the excess carrier charge in the dark $\left(d Q_{B} / d t\right)$, or (c) a combination of a time-dependent illumination and base charge (that is, a QSS measurement). Note that the variability of the SCR charge should be minimized, or corrected for. As a fourth method, one could think of (d) determining the effective lifetime from $d Q_{\mathrm{SCR}} / d t$ while suppressing $d Q_{B} / d t$, but the experimental conditions to achieve this usually correspond to the very low injection range, where the effective lifetime tends to be dominated by recombination in the SCR. Finally, it is possible to (e) obtain $\tau_{\text {eff }}$ by injecting a steady-state current in the dark, following that the intuitive interpretation of the total diode current is then equal to the total recombination within the device. Note that, even in nominally open circuit conditions, a current $J$ can flow as a consequence of a shunt resistance (if not already accounted for within the term $J_{\mathrm{SCR}}$ ) or to the loading of the measurement instrument, which can have a predominantly capacitive input impedance.

The importance of the rate of change of the base charge on effective lifetime measurements, $d Q_{B} / d t$, which corresponds to the base-region capacitance, has been discussed by Nagel et $a .^{3}$ in the context of photoconductance measurements and by Kerr et al. ${ }^{4}$ for voltage measurements. We focus here on the term $d Q_{\mathrm{SCR}} / d t$, which represents the capacitive effects occurring in the space-charge region. As the carrier injection level within the device varies due to changes in the excitation, the SCR charge also changes. For example, as the illumination is dimmed, the forward voltage across the 
junction decreases, and this has the effect of (a) reducing the excess carrier profile within the SCR and (b) modulating the width of the SCR, which expands towards its equilibrium value. The first of the two effects results in a decreasing recombination within the SCR, already included in the term $J_{\mathrm{SCR}}$ (which theoretically follows an ideality factor $m=2$ ). The second of the two is the predominant charge-storage effect that underlies the SCR capacitance. In response to a decreasing voltage, a slice of the quasineutral base region is converted into SCR (or vice versa, if the forward voltage increases). For this, carriers need to be extracted from (or injected into) the edges of the SCR. The corresponding current density can be approximately calculated using the common approximation of complete carrier depletion within the SCR. This approximation is more accurate for reverse than for forward bias, but is still acceptable for small values of the forward bias, which is the case we are interested in.

To reveal the main driving forces for the effect, we consider an asymmetric abrupt junction, where the dopant density in the emitter is much higher than that in the base region $\left(N_{E} \gg N_{B}\right)$. The charge due to the ionized dopant atoms in the SCR is approximately given by the following expression: ${ }^{13}$

$$
Q_{\mathrm{dopant}} \approx \sqrt{2 q \varepsilon_{S} N_{B}\left(\phi_{o}-V\right)} .
$$

The total number of electrons and holes that is required to compress the depletion region from its equilibrium (dark) value to that corresponding to the particular illumination (and bias voltage) under consideration is equal to and opposite in sign to the modification of the ionized dopant charge:

$$
Q_{\mathrm{SCR}}=\sqrt{2 q \varepsilon_{S} N_{B}}\left[\sqrt{\phi_{o}}-\sqrt{\left(\phi_{o}-V\right)}\right] .
$$

In a time-dependent situation (QSS, PCD, or OCVD), the charge $Q_{\mathrm{SCR}}$ varies with time at a rate:

$$
\frac{d Q_{\mathrm{SCR}}}{d t}=-\frac{d Q_{\mathrm{dopant}}}{d t} \approx \sqrt{\frac{q \varepsilon_{S} N_{B}}{2\left(\phi_{o}-V\right)}} \frac{d V}{d t},
$$

where $\varepsilon_{s}=\varepsilon_{0} \varepsilon_{r}$ is the permittivity of the semiconductor (for silicon $\left.\varepsilon_{r}=11.7\right), \phi_{o}$ is the electrostatic potential in equilibrium $\left(\phi_{o}=1-1.1 \mathrm{~V}\right.$ for typical silicon solar cells), and $V$ is the voltage that corresponds to a particular carrier injection level in the base. Alternatively, the relationship between the current due to the change of the SCR charge over time and the rate of change of the voltage can be expressed by means of the junction capacitance $C_{J}$ :

$$
\frac{d Q_{\mathrm{SCR}}}{d t}=C_{J}(V) \frac{d V}{d t} .
$$

Equation (9) indicates that the current, $d Q_{\mathrm{SCR}} / d t$, due to the modulation of the charge in the SCR is proportional to the square root of the base doping and to the rate of change of the voltage or, equivalently, of the carrier density. The distortion produced by the junction capacitance can, therefore, be expected to be greater for devices made on low-resistivity wafers and measurement conditions that result in a rapid change of the excess carrier density with time.

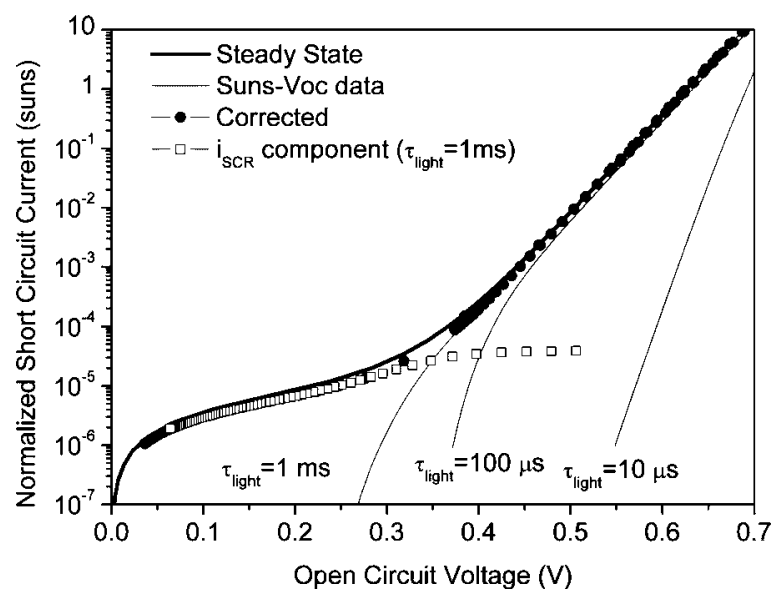

FIG. 1. Simulated illumination- $V_{\mathrm{oc}}$ characteristics of a silicon solar cell with a $p$-type base doping of $1.5 \Omega \mathrm{cm}$ and carrier lifetime $\tau_{\mathrm{nB}}=100 \mu \mathrm{s}$. The characteristics that would be obtained with three different light sources with dimming constants $\left(\tau_{\text {light }}=1 \mathrm{~ms}, 100 \mu \mathrm{s}\right.$, and $\left.10 \mu \mathrm{s}\right)$ are compared to the steady-state $I_{\mathrm{sc}}-V_{\mathrm{oc}}$ curve. The dots superimposed on the latter are the result of correcting the $\mathrm{QSSV}_{\mathrm{oc}}$ curves for junction capacitance effects.

\section{IMPACT OF JUNCTION CAPACITANCE ON QSS OPEN-CIRCUIT VOLTAGE MEASUREMENTS}

\section{A. Computer simulations}

With the help of the above equations, we can predict the effect of the SCR on QSS illumination- $V_{\mathrm{oc}}$ (that is, $\mathrm{QSSV}_{\mathrm{oc}}$ ) measurements, which are typically performed under a light source that changes slowly with time. Because of the junction capacitance and, depending on the voltage range and of the particular device, also of the base capacitive term $d Q_{B} / d t$, the voltage cannot respond instantly to the decreasing light intensity, and a higher than expected $V_{\text {oc }}$ will be measured. The computer simulations shown in Figs. 1 and 2 and the experimental measurements shown in Figs. 4 and 5 confirm that this is indeed the case.

Figure 1 presents computer simulations using the program $\mathrm{PC} 1 \mathrm{D}^{16}$ of a typical silicon solar cell with the following parameters: $p$-type base region with $N_{B}=10^{16} \mathrm{~cm}^{-3}, \tau_{\mathrm{nB}}$

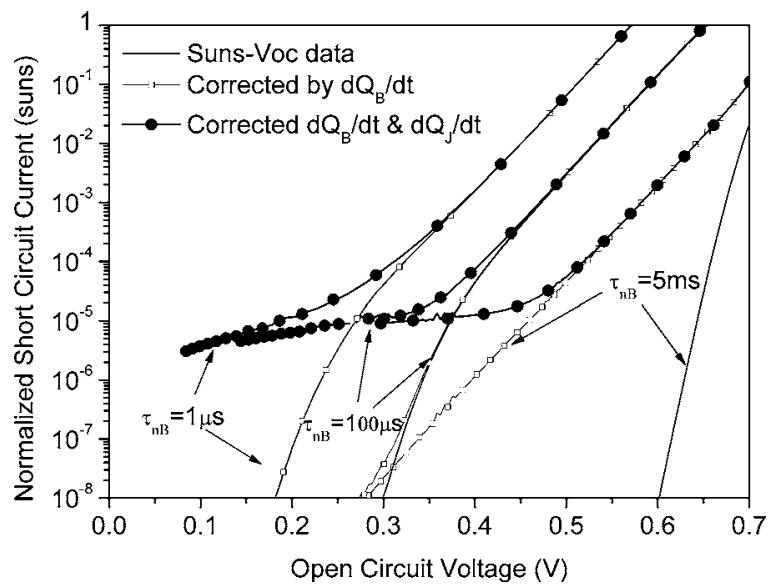

FIG. 2. Simulated illumination- $V_{\text {oc }}$ characteristics of three silicon solar cells with the same $p$-type base doping of $1.5 \Omega \mathrm{cm}$ but different carrier lifetimes of $10 \mu \mathrm{s}, 100 \mu \mathrm{s}$, and $5 \mathrm{~ms}$. The light source has a dimming constant of $\tau_{\text {light }}=1 \mathrm{~ms}$. The reconstruction of the static curves is shown in two steps: first the base charge correction and then the junction capacitance correction. 
$=100 \mu \mathrm{s}, S_{r}=100 \mathrm{~cm} / \mathrm{s}, W_{B}=150 \mu \mathrm{m}, N_{E}=10^{20} \mathrm{~cm}^{-3}, S_{f}$ $=10^{6} \mathrm{~cm} / \mathrm{s}, \quad W_{E}=1 \mu \mathrm{m} \quad\left(J_{\mathrm{oe}}=500 \mathrm{fA} / \mathrm{cm}^{2}\right), \quad J_{o_{(\mathrm{SCR})}}$ $=1 \mathrm{nA} / \mathrm{cm}^{2}$, and $G_{\mathrm{sh}}=10^{-6} \Omega \mathrm{cm}^{2}$. In addition to the true static $I-V$ device characteristics, we have simulated the opencircuit voltage $V_{\text {oc }}$ for three different characteristic time constants $\tau_{\text {light }}$ of the illumination (an exponential dimming has been assumed). A representative QSS light source typically has $\tau_{\text {light }}=1 \mathrm{~ms}$, which is ten times higher than the carrier lifetime of this particular device $\tau_{\mathrm{nB}}=100 \mu \mathrm{s}$. In such a case, the correcting terms due to the derivative of the base charge can be expected to be negligible, and (as can be seen in Fig. 1) the QSS $I-V$ characteristics are in excellent agreement with the steady-state ones for a broad range of voltages and illumination intensities. Nevertheless, at voltages below 0.37 $\mathrm{V}$, the QSS voltage (at a given illumination level) is considerably greater than the true steady-state one. The effect is similar in the case of a faster dimming of the illumination, with $\tau_{\text {light }}=100 \mu \mathrm{s}$, and becomes noticeable at even higher voltages $(0.45 \mathrm{~V}$, approximately).

Based on the theoretical considerations made above, the low voltage distortion of the QSS characteristics can be explained by the effect of the junction capacitance $C_{J}$. The issue now is whether it is possible to correct for the effect of $C_{J}$ in order to reconstruct the static $I-V$ characteristics. Taking the modeled voltage versus time as input data, we calculated the time-dependent current needed to charge the junction capacitance using Eq. (9). The result is plotted with open symbols in Fig. 1 for the case of $\tau_{\text {light }}=1 \mathrm{~ms}$. Subsequently, this SCR current was added to the QSS voltage versus illumination curve, resulting in the filled dots of Fig. 1. As can be seen, the resulting characteristic curve is almost perfectly superimposed on the static $I-V$ curve. A similarly good reconstruction of the static $I-V$ curve was obtained for the case of $\tau_{\text {light }}=100 \mu \mathrm{s}$.

A third quasitransient case with $\tau_{\text {light }}=10 \mu$ s corresponds quite closely to an OCVD situation. The main effect now is that of the charge in the base, whose rate of change is retarded by a carrier lifetime of $\tau_{\mathrm{nB}}=100 \mu \mathrm{s}$. Nevertheless, for voltages below $0.5 \mathrm{~V}$ (not plotted in Fig. 1), junction capacitance becomes dominant. Even in this range, typical of a transient decay in the dark, the simple model is capable of reconstructing the $I-V$ characteristics from the known voltage and its derivative as a function of time.

According to the modeling shown in Fig. 1, it is safest to use a light source that varies as slowly as possible, but even then junction capacitance effects may be noticeable in a certain range of voltages, depending on the relative value of the carrier lifetime of the material compared with the characteristic time of the illumination. Figure 2 shows additional computer modeling in order to explore different carrier lifetimes in the base region, from $\tau_{\mathrm{nB}}=1 \mu \mathrm{s}$ to an upper intrinsic limit of $\tau_{\mathrm{nB}}=5 \mathrm{~ms}$, using in all cases a typical QSS flash having $\tau_{\text {light }}=1 \mathrm{~ms}$. The device parameters are similar to those in Fig. $1\left(1.5 \Omega \mathrm{cm}, J_{o_{(\mathrm{SCR})}}=1 \mathrm{nA} / \mathrm{cm}^{2}\right.$, and $\left.G_{\mathrm{sh}}=10^{-6} \Omega \mathrm{cm}^{2}\right)$, except that the emitter and back surface recombination have been almost suppressed to emphasize the effects of the lifetime in the base $\left(N_{E}=10^{18} \mathrm{~cm}^{-3}, W_{E}=1 \mu \mathrm{m}, S_{f}=0 \mathrm{~cm} / \mathrm{s}\right.$, $J_{\mathrm{oe}}=16 \mathrm{fA} \mathrm{cm}^{-2}, S_{r}=0 \mathrm{~cm} / \mathrm{s}$, and $\left.W_{B}=150 \mu \mathrm{m}\right)$. If the car-

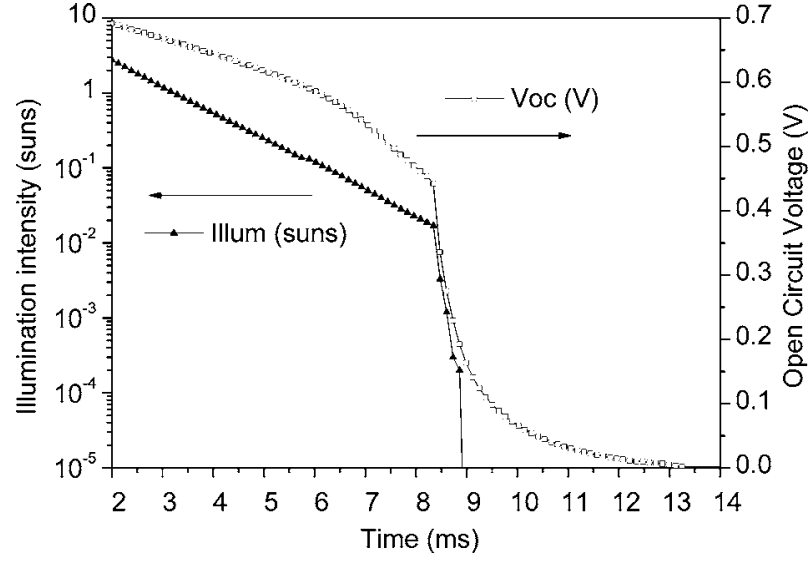

FIG. 3. Illumination intensity and open-circuit voltage for a $1 \Omega \mathrm{cm} p$-type silicon solar cell measured as a function of time. The first $8.5 \mathrm{~ms}$ corresponds to the quasi-steady-state regime (dimming characteristic constant $\tau_{\text {light }}=1.2 \mathrm{~ms}$ ), followed by a rapid drop in the light intensity (quasitransient regime, with $\tau_{\text {light cutoff }} \leqslant 100 \mu \mathrm{s}$ ), and finally a free decay of the open-circuit voltage in the dark (OCVD). The open-circuit voltage follows, for times greater than $9 \mathrm{~ms}$, the characteristic decay of a capacitance.

rier lifetime is very high $\left(\tau_{\mathrm{nB}}=5 \mathrm{~ms}\right)$ in comparison with the rate of change of the illumination $\left(\tau_{\text {light }}=1 \mathrm{~ms}\right)$, the device would be in quasitransient conditions and the main correction is the derivative of the base charge. Nevertheless, even in this case, correcting for the SCR capacitive effect is important, to accurately reconstruct the device characteristics at voltages below $0.45 \mathrm{~V}$. The other two cases analyzed $\left(\tau_{\mathrm{nB}}\right.$ $=100 \mu \mathrm{s}$ and $\tau_{\mathrm{nB}}=10 \mu \mathrm{s}$ ) are essentially static from the point of view of the base, and only the SCR capacitance needs to be corrected for. As can be seen in Fig. 2, the $C_{J}$ correction becomes significant at a higher voltage, the higher the bulk lifetime. This is a consequence of the horizontal voltage shift that results from the decreased recombination in the base.

\section{B. Experimental demonstration}

To demonstrate the effect, we have measured a $4 \mathrm{~cm}^{2}$ device made on a single-crystal silicon wafer having the usual resistivity for silicon solar cells, approximately $1 \Omega \mathrm{cm}$. To better explain the difference between the $\mathrm{QSSV}_{\mathrm{oc}}$ and OCVD methods, the measurement shown in Fig. 3 combines both. The first $8.5 \mathrm{~ms}$ is typical of the quasi-steadystate regime, obtained with a photographic flash with a dimming characteristic constant $\tau_{\text {light }}=1.2 \mathrm{~ms}$. This type of flash is not intended to have a very fast cutoff, and the graph in Fig. 3 indicates that $\tau_{\text {light cutoff }} \approx 100 \mu$ s, which represents a quasitransient regime. Finally, for times greater than $9 \mathrm{~ms}$, the light practically vanishes and the open-circuit voltage decays freely (OCVD), following the characteristic time response of a discharging capacitor.

The illumination- $V_{\mathrm{oc}}$ curve corresponding to the data given in Fig. 3 is plotted in Fig. 4. For comparison, the experimental dark $I-V$ curve is also shown as a continuous line. The $\mathrm{QSSV}_{\mathrm{oc}}$ method is directly accurate (that is, even without correction) only at voltages higher than $0.45 \mathrm{~V}$ for this particular illumination. Nevertheless, once corrected for the capacitive effects, the reconstructed QSSV ${ }_{\text {oc }}$ curve agrees very well with the static dark $I-V$ curve, even in the low 


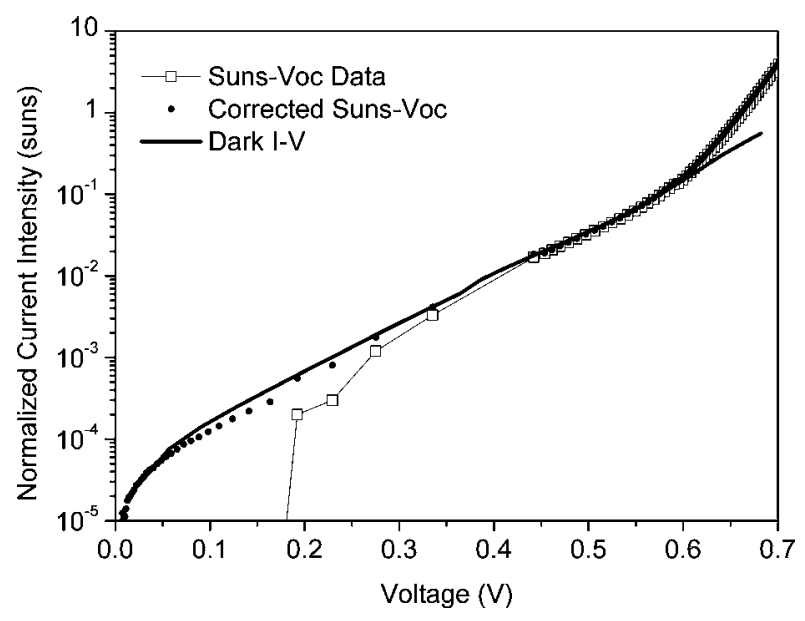

FIG. 4. Illumination- $V_{\mathrm{oc}}$ curve for the $1 \Omega \mathrm{cm}$ silicon solar cell measured as shown in Fig. 3 (open square symbols). The QSSV $_{\text {oc }}$ method is directly accurate only at voltages higher than $0.45 \mathrm{~V}$ for this particular illumination. The reconstructed curve, given by the closed symbols, includes the OCVD range of the measurement, dominated by junction capacitance effects. For comparison, the experimental dark $I-V$ curve is shown as a continuous line.

voltage range. Remarkably, the successful reconstruction of the curve includes the data points corresponding to the OCVD range of the measurement. Although two-thirds of the capacitive effects could be explained by the simple model of Eq. (9), in this occasion the input capacitance of the instrument (an A/D card) also played a noticeable role. This external loading capacitance was measured to be $80 \mathrm{nF}$, while the solar cell had a junction capacitance in the range of $160 \mathrm{nF}$. The latter was confirmed by measuring the $C-V$ curve in reverse bias, ${ }^{17}$ which allowed a more precise determination of the base doping $(0.8 \Omega \mathrm{cm})$. The model, taking into account both the junction capacitance and the impedance of the measuring equipment, permits a complete reconstruction of the static illumination- $V_{\text {oc }}$ curve from this combined QSS and OCVD measurements.

The simulations presented in Fig. 2 showed the relevance of the junction capacitance effect as a function of the speed at which the excitation changes and the excess carriers recombine. In addition, the effect of $C_{J}$ also depends strongly on the base doping. As described by Eq. (9), it approximately increases with the square root of the base dopant density. To illustrate this, we have selected a solar cell made on a $0.1 \Omega \mathrm{cm}$ silicon wafer and measured it entirely using the $\mathrm{QSSV}_{\text {oc }}$ method over several decades of light intensity, which were achieved with six combinations of neutraldensity filters and a photographic flash having an approximate $\tau_{\text {light }}=1.2 \mathrm{~ms}$. The calibrated detector used to measure the light intensity also had six ranges of sensitivity. The resulting illumination- $V_{\text {oc }}$ characteristics are shown in Fig. 5, together with the static $I-V$ characteristics, which were measured in the dark, point by point. The distortion produced by the junction capacitance is obvious at voltages below $0.4 \mathrm{~V}$. Next, we have applied the simple model of Eq. (9) to calculate the equivalent steady-state illumination (that is, the actual illumination intensity plus the SCR current $d Q_{\mathrm{SCR}} / d t$ ) corresponding to every voltage. The result is a corrected

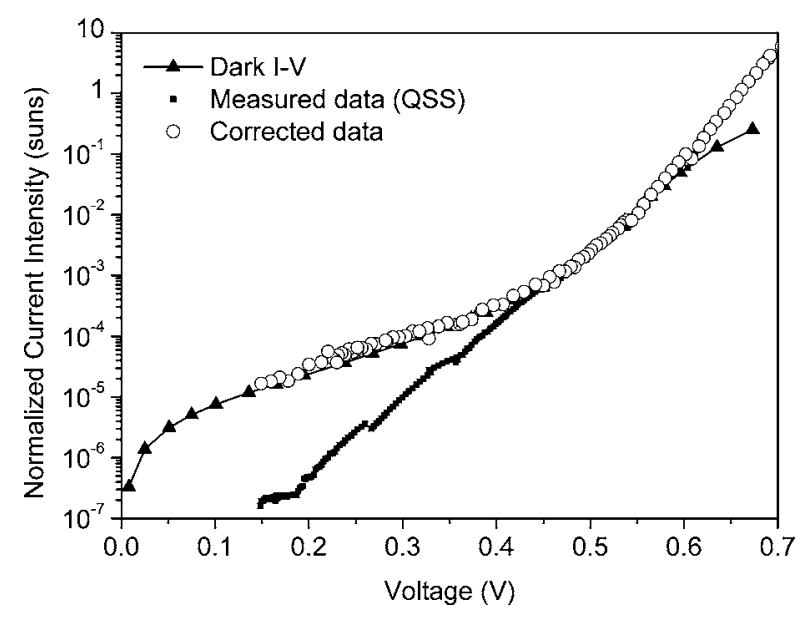

FIG. 5. Experimental dark $I-V$ curve, QSS illumination- $V_{\mathrm{oc}}$ data, and reconstructed $I-V$ curve for a $0.1 \Omega \mathrm{cm} p$-type silicon solar cell. The QSS illumination- $V_{\text {oc }}$ curve was measured using several flashes with a dimming constant of $\tau_{\text {light }}=1.2 \mathrm{~ms}$.

illumination- $V_{\text {oc }}$ curve that agrees very well with the dark $I-V$ one, thus proving the validity of the model.

It is obvious from Eq. (9) that, to be able to correct for the effects of junction capacitance, it is necessary to know the dopant density in the base and the value of the builtin equilibrium potential difference across the $p n$ junction. Fortunately, these parameters do not need to be accurately known, for example, a $10 \%$ error in $N_{B}$ or in $\phi_{o}$ results in less than $6 \%$ error in the evaluation of the junction capacitive current. Nevertheless, $N_{B}$ can be easily determined from measurements of the junction capacitance in reverse or moderate forward bias. ${ }^{17}$

\section{THE EFFECT OF JUNCTION CAPACITANCE ON LIFETIME MEASUREMENTS}

To assess the possible impact of junction capacitance on lifetime measurements it is useful to express the current needed to charge it directly as a function of the carrier density. In low injection, Eq. (9) can be simplified to

$$
\frac{d Q_{\mathrm{SCR}}}{d t} \approx \sqrt{\frac{k T N_{B} \varepsilon_{S}}{2 \ln \left[N_{E} / \Delta n(0)\right]}} \frac{1}{\Delta n(0)} \frac{d[\Delta n(0)]}{d t} .
$$

Note that in this expression, the excess carrier density is to be calculated at the edge of the SCR $(x=0)$, which can, in many cases, be substantially different to the average density in the base region.

Using the computer simulation program PC1D, ${ }^{14}$ we have calculated the excess carrier density as a function of time and illumination in a few representative cases. Figure 6 shows the simulated effective lifetime as a function of the average excess carrier density in the base $\Delta n$ that corresponds to the solar cell in Fig. 1, with a $p$-type base region having $N_{B}$ $=10^{16} \mathrm{~cm}^{-3}$ and $\tau_{\mathrm{nB}}=100 \mu \mathrm{s}$. Note that the highest value of the effective lifetime is only $\tau_{\text {eff }}=24 \mu \mathrm{s}$, due to the limits imposed by the front emitter and the rear surface. The two extreme illumination cases of quasistatic $\left(\tau_{\text {light }}=1 \mathrm{~ms}\right)$ and quasitransient $\left(\tau_{\text {light }}=10 \mu \mathrm{s}\right)$ conditions have been analyzed. If a proper QSS illumination is used $\left(\tau_{\text {light }}=1 \mathrm{~ms}\right)$, the effect of the junction capacitance is almost negligible for this de- 


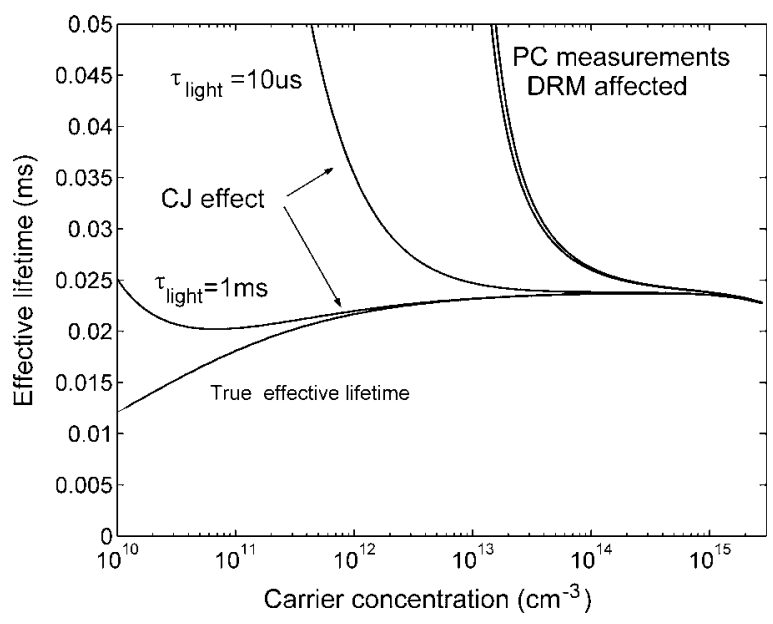

FIG. 6. Effective lifetime as a function of average excess carrier density in the base for the same device used in Fig. 1. Computer simulations include techniques based on voltage or luminescence measurements, which are only affected by the junction capacitance and techniques based on photoconductance measurements, where the depletion region modulation effect is dominant.

vice. It does become dominant, however, in the quasitransient situation $\left(\tau_{\text {light }}=10 \mu \mathrm{s}\right)$ at carrier injection levels below $10^{13} \mathrm{~cm}^{-3}$.

The effects of junction capacitance on lifetime measurements are, therefore, only significant if the technique used to determine the excess carrier density is very sensitive. Photoluminescence has recently been shown to reach down to the $10^{11} \mathrm{~cm}^{-3}$ range. ${ }^{5}$ Even lower excess carrier densities can be easily reached from voltage measurements such as $\mathrm{QSSV}_{\mathrm{oc}}$. The excess carrier density at the edge of the junction spacecharge region can be determined from the voltage:

$$
n p \approx \Delta n(0)\left[N_{B}+\Delta n(0)\right]=n_{i}^{2} \exp \frac{q V_{\mathrm{oc}}}{k T},
$$

where $n_{i}^{2}$ is the $p n$ product in equilibrium and, for a $p$-type base region, $n \approx \Delta n$ and $p \approx N_{A}+\Delta n$. It is, therefore, possible to convert illumination- $V_{\mathrm{oc}}$ curves into effective lifetime versus carrier density curves, as illustrated by the correspondence between Figs. 1 and 6 .

When the magnitude being measured is the excess photoconductance of the wafer, a much stronger effect makes its appearance, the modulation of the width of the depletion region or DRM. ${ }^{7-10}$ It stems from the compression of the depletion region from its equilibrium value to that corresponding to the particular injection level under consideration, ${ }^{10}$ or vice versa. This requires an additional number of holes and electrons, given by $Q_{\mathrm{SCR}}$ in Eq. (8), which do not participate in the recombination processes, since they are separated by the junction. These extra electrons and holes are generally mobile, and contribute to the conductance of the wafer. The total excess photoconductance is given by the sum of the contributions due to the carrier charge within the base and in the SCR:

$$
\Delta \sigma=\sigma_{L}-\sigma_{\mathrm{dark}} \approx Q_{B}\left(\mu_{n}+\mu_{p}\right)+Q_{\mathrm{SCR}}\left(\mu_{n}^{\prime}+\mu_{p}\right) .
$$

For simplicity, we have assumed that the excess electron and hole densities within the quasineutral base region are identi- cal, $\Delta n=\Delta p$, and that their mobilities are spatially constant. The second term of the right-hand side represents the DRM effect, which becomes noticeable only at very low injection levels, when $Q_{B}<Q_{\mathrm{SCR}}$. If this additional contribution to the photoconductance is misinterpreted as being part of the base charge, an abnormally high effective lifetime is determined (as shown in Fig. 6). Note that the two sides of the junction store an identical (albeit opposite in sign) charge $Q_{\mathrm{SCR}}$. We have indicated in Eq. (13) that, in the $n^{+}$emitter diffusion side, the mobility of electrons can be expected to be lower than in the lowly doped base region. Similarly, $n^{+}$regions can be induced in $p$-type silicon wafers by certain dielectric layers that contain a strong positive charge, including $\mathrm{SiO}_{2}$ and $\mathrm{SiN}$. The mobility of electrons in such inversion layers has been shown to be significantly reduced. ${ }^{7}$ This is, nevertheless, only a relatively minor refinement in the modeling of the DRM effect. Note that in forward bias there is, in addition to $Q_{\mathrm{SCR}}$, a small excess concentration of electrons and holes within the SCR that gives rise to the well-known SCR recombination current.

It is evident that the DRM effect and the junction capacitive effect discussed in this paper are closely related, since both originate in the SCR charge. Previous works, ${ }^{7-10}$ only dealt with the impact of the DRM on the photoconductance. It is important to note that the DRM effect is present either in transient PCD experiments ${ }^{7}$ or under quasistatic ${ }^{10}$ or fully static illumination. Indeed, it can be observed to be identical for the two illumination conditions studied in Fig. 6. The SCR capacitive effect, on the other hand, stems from the rate of change of $Q_{\mathrm{SCR}}$ with time, and does not affect fully static measurements.

Figure 6 shows that the DRM artifact is dominant at carrier densities below $10^{14} \mathrm{~cm}^{-3}$ for this representative solar cell wafer. Therefore, junction capacitance effects are unlikely to be found in lifetime measurements unless a nonphotoconductive technique is used, such as luminescence or voltage, and the sensitivity of the instruments is sufficient to reach very low carrier densities. Lifetime measurements based on photoluminescence ${ }^{5}$ have been shown to be exempt from DRM effects and pointed out to be proportional to the separation between electron and hole quasi-Fermi levels, which are equivalent to measuring the voltage. It is to be expected that transient or quasitransient photoluminesce measurements will be subject to junction capacitance effects, just like QSSV $_{\text {oc }}$ measurements. This provides a qualitative explanation for the observation by Trupke et al. ${ }^{18}$ that the lifetime measured at low carrier densities by transient photoluminescence was higher than that measured by quasisteady-state photoluminescence. In addition to DRM, carrier trapping ${ }^{19}$ at shallow energy levels together with experimental noise frequently dominate the ultralow injection range.

\section{CONCLUSIONS}

Our analysis of the transient phenomena that may be present in quasi-steady-state (QSS) measurements has revealed that the capacitance of the space-charge region has an important effect at low carrier injection levels. This has clear repercussions on QSS measurements of illumination- $V_{\mathrm{oc}}$ 
characteristic curves. Even if a slowly changing illumination is used to ensure nearly static conditions from the point of view of the base region, junction capacitance effects can still be predominant at low voltages.

Fortunately, the customary analytical model for the junction capacitance permits to obtain the static illumination- $V_{\mathrm{oc}}$ characteristics from time-dependent measurements in a simple manner. This model has been verified by means of computer simulations and experimental measurements. In all the cases analyzed here, which ranged from transient decay to almost fully static conditions, we found an excellent agreement between the reconstructed illumination- $V_{\text {oc }}$ characteristics and the true steady-state ones. In practice, real solar cells frequently have a low shunt resistance, which would present an easier path for the discharge of the junction capacitance, and hence reduce its impact. Therefore, the effect of $C_{J}$ is more likely to be observed in high-quality cells.

\section{ACKNOWLEDGMENTS}

This work has been funded by the Australian Research Council. The research of one of the authors (F.R.) at the ANU has been possible thanks to the Basque Government's initiative Programas de Perfeccionamiento y Movilidad de Personal Investigador.

${ }^{1}$ R. A. Sinton and A. Cuevas, Proceedings of the 16th European Photovoltaic Solar Energy Conference, Glasgow, Scotland, 1-5 May 2000 (James and James, London).

${ }^{2}$ R. A. Sinton and A. Cuevas, Appl. Phys. Lett. 69, 2510 (1996).
${ }^{3}$ H. Nagel, C. Berge, and A. G. Aberle, J. Appl. Phys. 86, 6218 (1999). ${ }^{4}$ M. J. Kerr, A. Cuevas, and R. A. Sinton, J. Appl. Phys. 91, 399 (2002).

${ }^{5}$ T. Trupke and R. A. Bardos, Proceedings of the 31st Photovoltaic Specialists Conference and Exhibition, Orlando, Florida, 3-7 January 2005 (IEEE, New York), 903.

${ }^{6}$ L. Castañer, J. Phys. D 14, 1867 (1981).

${ }^{7}$ O. Hahnheiser, L. Elstner, S. Von Aichberger, and M. Kunst, Proceedings of the Second World Conference on Photovoltaic Solar Energy Conversion, Vienna, Austria, 6-10 July 1998 (European Commission, Ispra, Italy).

${ }^{8}$ M. Bail, M. Schulz, and R. Brendel, Appl. Phys. Lett. 82, 757 (2003).

${ }^{9}$ D. H. Neuhaus, P. J. Cousins, and A. G. Aberle, Proceedings of the Third World Conference on Photovoltaic Solar Energy Conversion, Osaka, Japan, 12-16 May 2003 (WCPEC-3 Organizing Committee, Osaka, Japan).

${ }^{10}$ P. J. Cousins, D. H. Neuhaus, and J. E. Cotter, J. Appl. Phys. 95, 1854 (2004).

${ }^{11}$ H. K. Gummel and H. C. Poon, Bell Syst. Tech. J. 49, 827 (1970).

${ }^{12}$ T. Jung and F. Lindholm, IEEE Trans. Electron Devices 31, 588 (1984).

${ }^{13}$ R. M. Warner and B. L. Grung, Semiconductor-Device Electronics (Holt, Rinehart and Winston-Saunders College Publishing, Philadelphia, 1991).

${ }^{14}$ A. Cuevas, M. Stocks, D. Macdonald, and R. Sinton, Proceedings of the Second World Conference on Photovoltaic Solar Energy Conversion, Vienna, Austria, 6-10 July 1998 (European Commission, Ispra, Italy).

${ }^{15}$ A. Cuevas and R. Sinton, in Practical Handbook of Photovoltaics: Fundamentals and Applications, edited by T. Markvart and L. Castaner, (Elsevier, Oxford, 2003).

${ }^{16}$ P. A. Basore, IEEE Trans. Electron Devices 37, 337 (1990).

${ }^{17}$ F. Recart and A. Cuevas, Proceedings of the 20th European Photovoltaic Solar Energy Conference, Barcelona, Spain, 6-10 June 2005 (unpublished).

${ }^{18}$ T. Trupke, R. A. Bardos, F. Hudert, P. Würfel, J. Zhao, A. Wang, and M. A. Green, Proceedings of the 19th European Photovoltaic Solar Energy Conference, Paris, France, 7-11 June 2004 (WIP-Munich and ETAFlorence)

${ }^{19}$ D. Macdonald and A. Cuevas, Appl. Phys. Lett. 74, 1710 (1999). 